\title{
The Advanced Gamma Ray Tracking Array AGATA
}

\author{
C. Rossi Alvarez \\ Dipartimento di Fisica and INFN, Sezione di Padova, I-35131 Padova, Italy
}

Received on 3 November, 2003

\begin{abstract}
New accelerator facilities for radioactive-ion beams and high-intensity stable beams will start operation in a few years. Although these beams will provide interesting opportunities for exploring unknown territories of the nuclear landscape, the experimental conditions will be very challenging and, indeed, the nuclear structure community has realized that a new generation of powerful arrays for $\gamma$-ray spectroscopy has to be built in order to cope with them. As a result of years of experience with Compton suppressed germanium arrays and of intensive R\&D work targeted to extend their limits, it is now clear that the next $4 \pi \gamma$-ray spectrometers will be built fully from germanium detectors and will be based on the technique of $\gamma$-ray tracking. The "Advanced GAmma Tracking Array" (AGATA), proposed in Europe, will be an instrument of major importance for nuclear structure studies at the very limits of nuclear stability. It will be built out of 120/180 highly segmented Ge crystals operated in position sensitive mode by means of digital data techniques and pulse shape analysis of the segment signals. AGATA will be able to measure $\gamma$ radiation in a large energy range (from $\sim 10 \mathrm{keV}$ to $\sim 10$ $\mathrm{MeV})$, with the largest possible photopeak efficiency $\left(25 \%\right.$ at $\left.M_{\gamma}=30\right)$ and with a good spectral response. In particular, its very good Doppler correction and background rejection capabilities will allow to perform "standard" $\gamma$-ray spectroscopy experiments using fragmentation beams with sources moving at velocities up to $\beta \sim 0.5$.
\end{abstract}

\section{Introduction}

In the last decade the most advanced nuclear structure studies have been carried out using large Germanium detector arrays like EUROBALL and GAMMASPHERE. These arrays have the highest efficiency so far available for in-beam $\gamma$-ray spectroscopy. They are built out of $\sim 100$ Comptonsuppressed high-purity germanium (HPGe) spectrometers arranged in a spherical configuration around the reaction point and provide, for $1 \mathrm{MeV} \gamma$-rays, a total peak efficiency $\epsilon_{p h}$ of about $10 \%$ and a P/T-ratio of about $60 \%$.

The $\gamma$-ray spectroscopy community is offered now the opportunity to extend the field of nuclear structure studies exploiting the radioactive ion beams provided by the next generation of accelerator facilities of ISOL and fragmentation type. It is well realized however that, due to low beam intensities and large Doppler broadenings combined with high backgrounds, the experimental conditions at such facilities will be very challenging. To cope with them, both the total peak efficiency and the selectivity of our detector arrays must be improved, However such an array cannot be obtained by simply increasing the number of detectors. The reason is that to obtain a good $\mathrm{P} / \mathrm{T}$ ratio we must surround the Ge crystals with BGO suppression shields which, however, take a consistent fraction of the solid angle and limit the total peak efficiency of the so-called $4 \pi$ arrays to no more than $10 \%$. Recently, it was realized that more could be gained by adding back the Compton scattered $\gamma$-ray rather than using it to suppress the original $\gamma$-ray, but for this purpose one needs to know the $\gamma$-ray trajectory within the Ge.

One possible solution of this problem may come from recent advances in crystal segmentation technology and digital signal processing, which make it possible to operate the germanium detectors in a position sensitive mode. The knowledge of energy, position and time of the interaction points within the germanium crystal allows to reconstruct the interaction history and time correlations of the absorbed gamma radiation, leading to the so-called gamma-ray tracking concept [1].

The tracking allows to build a compact array solely out of highly segmented Ge. As it is expected from simulations, an array consisting of a limited number (100-200) of such detectors could have an efficiency of up to $50 \%$ and a P/Tratio of $70 \%$. It should be remarked that an important benefit of $\gamma$-ray tracking is the possibility to determine with very good precision $\left(\sim 1^{\circ}\right)$ the emission direction of the reconstructed transitions, allowing for an almost perfect Doppler broadening correction for gammas emitted by nuclei recoiling at velocity as high as $v / c=0.5$.

Finally, it is clear that, besides the perspective use for fundamental research, the principle of $\gamma$-ray tracking is also extremely interesting for its possible application in the field of $\gamma$-ray imaging.

\section{Gamma-ray tracking methods}

The feasibility of $\gamma$-ray tracking has been the subject of extensive R\&D performed in the last 7 years by GRETA [2] in the USA and the TMR network "Gamma Ray Tracking Detectors"[3] in Europe. The main lines of development are outlined in the following. 


\subsection{Tracking algorithms.}

The task of $\gamma$-ray tracking is to identify the individual $\gamma$ rays and reconstruct their scattering sequence in the active volume of the detector from the knowledge of energy and spatial coordinates of the interaction. As long as single $\gamma$ events are considered, the characteristic features of the relevant interaction mechanisms can be exploited for the task of $\gamma$-ray tracking in simple and efficient ways.

Isolated low energy interaction points can be accepted as photoelectric absorption events upon checking the compatibility between energy and interaction depth in the detector.

In the intermediate energy range the $\gamma$-rays undergo a sequence of Compton scatterings with a final photoelectric effect. For the reconstruction of such events, a figure of merit of each Compton scattering vertex (Fig. 1) is calculated for all permutations of the interaction points. The figure of merit is defined as the deviation between the measured energy of the interaction point and the energy calculated from the Compton formula (using the scattering angle derived from the spatial position of the involved points).

The permutation with the best total merit is considered to correspond to the real scattering sequence and the event is accepted if its value is compatible with predefined limit.

For $\gamma$-ray energies above a few $\mathrm{MeV}$, pair production events become important. As long as shower effects are negligible a strong signature of this mechanism is that the first point of interaction collects the total $\gamma$-ray energy minus the $2 m_{0} c^{2}$ needed to create the pair. This is because the electron and positron are absorbed within a distance of $\sim 1 \mathrm{~mm}$, while the two annihilation photons generate other clusters of points somewhere else in the array where they are possibly detected. A simple algorithm built to exploit these features has a reconstruction efficiency of about $50 \%$.

The tracking algorithms mentioned above have been developed and tested using data from Monte Carlo simulations. In order to be realistic, the calculated interaction points are randomly smeared, before being used by the tracking algorithms, so as to take into account the position and energy resolution of actual detectors. It is important to remark that, due to experimental uncertainties but also because of fundamental limits, each of these algorithms will always accept some background events (corresponding e.g. to partial energy release in the detector) and reject "good" ones. The amount of accepted background can often be reduced, but this always at the cost of an increased rejection of good events: i.e. a better P/T implies a lower efficiency.

For realistic events with several coincident $\gamma$-rays, one cannot simply apply the same considerations because there is no absolutely reliable way to determine the transition to which the individual interaction points belong. In principle, one could try all the possible ways to combine the measured points into a reasonable number of transitions but this is an extremely difficult computational task because already for medium $\gamma$-ray multiplicities the number of partitions can easily exceed $10^{20}$. On the other side, it does not really make sense to try it because the limited capability of the reconstruction algorithms to distinguish good events from bad ones (e.g. points of different transitions accepted as belonging together) does not ensure that the optimum partition can be identified. The problem has therefore to be split into simpler pieces and this can be achieved in at least two ways. Using the fact that the interaction points of transitions emitted into sufficiently separated directions tend to cluster into spatially isolated groups, it is possible to search for such cluster and validate them as individual transitions with the methods explained above. An example of a reconstructed high multiplicity event detected by an ideal germanium shell is shown in on the right part of Fig. 1. With a position resolution of 5 mm, this "ideal shell" yields $\epsilon_{p h}=36 \%$ and $\mathrm{P} / \mathrm{T}=60 \%$, for $\mathrm{M}_{\gamma}=30$.

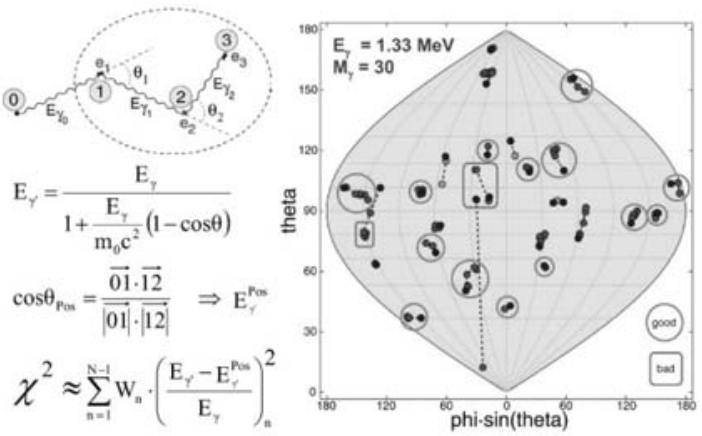

Figure 1. The left part shows the ingredients of $\gamma$-ray racking of a $\gamma$-ray absorbed in the detector material by means of two Compton scattering events followed by a photoelectric interaction. The right part shows the reconstruction of a high multiplicity event detected in an ideal germanium shell with an inner radius of $15 \mathrm{~cm}$ and a thickness of $9 \mathrm{~cm}$. Of the 30 emitted gammas 27 are detected; 23 of them with full energy release. The tracking algorithms recognized correctly 14 gammas, corresponding to $\sim 70 \%$ reconstruction efficiency. The two badly reconstructed groups of points end up into the spectrum background.

Slightly smaller reconstruction efficiencies are achieved with another approach, called "backtracking" [4], which starts from points with energy in the $\sim 100 \mathrm{keV}$ range (likely to be the last, i.e. photoelectric, interaction of a transition) and goes back, step by step, to the origin of the incident $\gamma$-ray looking for the correct Compton scattering vertices. Backtracking is probably more sensitive to position errors but is better suited for $\gamma$-ray imaging purposes.

\subsection{Highly segmented germanium detectors.}

Several laboratories are pursuing the development of highly segmented germanium detectors, both of the cylindrical and the planar configuration. The following brief summary is limited to closed-end coaxial detectors, which are believed to be more suited for the construction of $4 \pi$ tracking arrays. The 36-fold segmented "hexaconical" detector for the $4 \pi$ array GRETA was the first prototype to be studied in details [5] at Berkeley. In Italy, within the MARS collaboration, we are using a 25 -fold segmented detector that has 6 angular sectors, 4 transversal slices and a small segment on the front face [6]. To achieve the best energy resolution, these two detectors use cold FETs but there also prototypes 
of segmented detectors that use simpler to handle room temperature FETs that can be placed outside the ge-crystal vacuum chamber. Two such detectors (one with 24 and one with 36 segmented) under test at the University of Liverpool and Surrey, in the UK. At NSCL, the array SeGA composed of eighteen 32-fold-segmented cylindrical detectors, also with warm FETs, has just entered into operation. This array is not capable of fully tracking the detected gammas; instead, the high segmentation of the germanium detectors are used to improve the energy resolution in low multiplicity experiments with fast exotic beams.

\subsection{Pulse shape analysis (PSA).}

Necessary input data for the tracking algorithms are, for each point of interaction, three-dimensional position and deposited energy. This information is obtained from the analysis of the shapes of the signals from the segments. A characteristic feature of segmented detectors is that not only the segment that collects the net charge released by the interaction has a signal but also the neighbouring segments have transient signals with no net charge at the end. Their amplitudes increase with decreasing distance between the interaction point and the border to the neighboring segment. Using finite element analysis, signal shapes can be calculated accurately for almost arbitrary crystal geometries [7].

To test the algorithms for pulse shape analysis, simplified problems considering one or two interactions randomly distributed within only one segment are treated at present. The resulting composite signals from the segments are analyzed (decomposed) to extract the number, position, and energy of the original point(s) in various ways. Programs using a genetic algorithm (GA) determine the number of interactions correctly for $89 \%$ of the events. The variance between reconstructed and true position is $\sim 2 \mathrm{~mm}$ while that of the energies is $\sim 4 \%$.

The performance of the PSA algorithms has been tested with experimental data from the GRETA and MARS prototypes using tightly collimated $\gamma$-sources.

\subsection{Digital electronics and DAQ.}

To preserve the full signal-shape information that is needed to extract energy, timing and position of the interaction points, the electronics has to sample the preamplifier signals with fast ADCs located as close as possible to the detectors. It is not yet clear whether full pulse shape processing can be done in real time at the detector level. However, in order to reduce the amount of information transferred to the data acquisition system to a manageable value, one should be able to obtain at least a local trigger and the energy of the fired segments using the so-called Moving Window Deconvolution (MWD) [8]. This information and the subset of samples relative to the leading edge of the signals will be time stamped and transferred by high-speed fiber links to the data acquisition computers. It is foreseen that the overall data flow to the DAQ of a $4 \pi \gamma$-ray tracking array can be in excess of 500 Mbytes/s. Finally, after applying all necessary functions of data merging and gain matching, the pulse shape analysis will be performed and the tracking algorithms will be applied to produce "standard" events containing just energy, time and spatial direction of the reconstructed transitions.

\section{AGATA}

\subsection{The Array.}

The Advanced GAmma Tracking Array (AGATA) [9] project is aimed at a full scale implementation of the $\gamma$-ray tracking concept in a European context. A similar project is pursued in the USA by the GRETA [2] collaboration. The specific event that triggered the development of AGATA is the availability, in the near future, of radioactive beams from the upgraded GSI facility, from GANIL and from the SPES facility at LNL. However, the detector is being designed for a more general range of applications, e.g. also for experiments with high intensity stable beams.
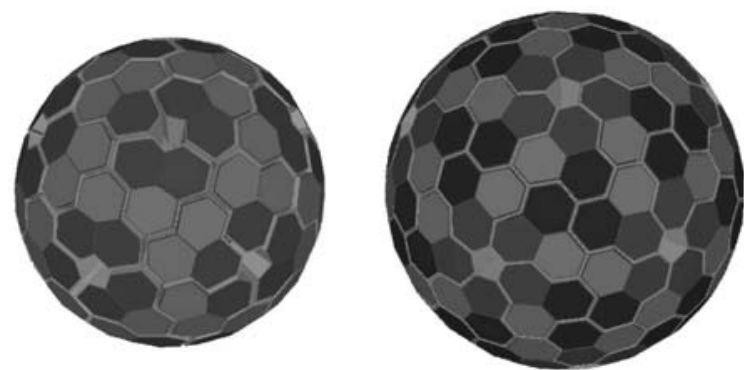

Figure 2. Schematic view of the two possible AGATA configurations. The smaller configuration has 120 hexagonal crystals of 2 different shapes (colour coded) that can be packed into 40 triple clusters of two different types. In the larger configuration 180 crystals of three different shapes are packed into 60 all-equal clusters.

As already mentioned, specific features of radioactive beams are limited intensities, (particularly for the most exotic nuclei); wide range of recoil velocities (from stopped to $v / c \sim 50 \%$ ); high gamma and particle backgrounds and $\gamma-$ ray multiplicities up to $M_{\gamma}=30$. To cope with these conditions, a $4 \pi \gamma$-array with the highest efficiency, selectivity, energy resolution and capability to handle high counting rates is required. These features can only be achieved with a close packed arrangement of germanium detectors, i.e. a $4 \pi$ shell built from large, highly segmented Ge crystals. The geometric structure of AGATA is based on a geodesic tiling of the spherical surface with hexagons and 12 pentagons. Two possible configurations have been identified (see Fig. 2): a smaller one with 120 hexagons (two different shapes) and a bigger one with 180 hexagons (three different shapes). The pentagons will, most likely, not be used and, to minimize inter-detector space losses, the hexagons will be packed into clusters of three crystals. The 120 configuration will therefore have 40 clusters (of two different types) while the 180 configuration will have 60 all-equal clusters. Using the largest reasonably available germanium crystals $(80 \mathrm{~mm}$ diameter, $90 \mathrm{~mm}$ length), the inner radius of the two arrays turns out to be 18 and $23 \mathrm{~cm}$, while the total solid angle covered by germanium is $\sim 72 \%$ and $\sim 80 \%$ respectively. 
The performance of the two candidate arrays has been simulated within the GEANT4 [10] framework including, for the sake of being realistic, the encapsulation of the crystals and their canning into triple clusters [11]. In Tab. 1 the obtained total peak efficiency and peak to total ratio are compared with the performance of the "ideal shell" and of EUROBALL. Even if a realistic detector can achieve only about $50 \%$ of the performance of the ideal shell, the efficiency gain with respect to EUROBALL is really considerable. In particular for high $\gamma$-multiplicity experiments, this means an increase in selectivity of several orders of magnitude.

The germanium crystals will be 36 -fold segmented and the resulting 4440 or 6660 total segments will provide unprecedented position sensitivity. A key feature of AGATA is the high precision for determining the emission direction of the detected $\gamma$-rays of $<1^{\circ}$ (corresponding to an effective solid angle granularity of more than 5104 that cannot be realistically achieved with individual germanium crystals). This granularity ensures an energy resolution better than $0.5 \%$ for transitions emitted by nuclei recoiling at velocities as high as $50 \%$ of the speed of light. This value is only a factor of two bigger than the intrinsic resolution of $\mathrm{Ge}$ detectors and is comparable with the values currently observed at 10 times smaller recoil velocity.

TABLE I. Performance of the possible AGATA configurations at $\mathrm{E}_{\gamma}=1 \mathrm{MeV}$

\begin{tabular}{lcccc}
\hline Config. & $\begin{array}{c}\text { Number of detectors } \\
\text { (cristals) }\end{array}$ & $\begin{array}{c}\text { Amount of } \\
\text { Germanium }(\mathrm{kg})\end{array}$ & $\begin{array}{c}\epsilon_{p h}[\mathrm{P} / \mathrm{T}] \% \\
\mathrm{M}_{\gamma}=1\end{array}$ & $\begin{array}{c}\epsilon_{p h}[\mathrm{P} / \mathrm{T}] \% \\
\mathrm{M}_{\gamma}=30\end{array}$ \\
\hline Ideal $4 \pi$ shell & 1 & 233 & $65[85]$ & $36[60]$ \\
\hline AGATA 120 & $40(120)$ & 212 & $33[54]$ & $19[44]$ \\
\hline AGATA 180 & $60(180)$ & 320 & $38[53]$ & $24[44]$ \\
\hline EUROBALL & $71(239)$ & 210 & $9[56]$ & $6[37]$ \\
\hline
\end{tabular}

\subsection{The demonstrator.}

According to the recently signed MoU, the development of AGATA will proceed in stages with a final R\&D phase preceding the construction of the full array. The objective of the $R \& D$ phase is to build a subsystem of 5 triple-clusters, called the AGATA demonstrator. The demonstrator, complete of digital electronics, DAQ and full on-line processing of the digitized data, will be used for testing the $\gamma$-ray tracking concept in real experiments.

\section{Acknowledgments}

The reported work is the result of a collaboration of European laboratories carried on originally within TMR Research Network "Development of $\gamma$-Ray Tracking Detectors for $4 \pi \gamma$-Ray Arrays" which was supported by the EC under contract ERBFMRX-CT97-0123.

\section{References}

[1] I.Y. Lee, Nucl. Instr. Meth. A422, 195 (1999).

[2] M.A. Deleplanque et al., Nucl. Instr. Meth. A430, 292 (1999).

[3] R.M. Lieder et al., Nucl. Phys. A682 279c (2001).

[4] J. van der Marel and B. Cederwall, Nucl. Instr. Meth. A437, 538 (1999).

[5] K. Vetter et al., Nucl. Instr. Meth. A452, 105 and 223 (2000).

[6] D. Bazzacco et al., LNL Ann.Rep 2000, p 166, LNLINFN(Rep)-178/01.

[7] Th. Krö and D. Bazzacco, Nucl. Instr. Meth. A463, 227 (2001).

[8] A. Georgiev et al., IEEE trans. Nucl. Sci. 41, 1116 (1994).

[9] http://agata.pd.infn.it/documents/Agata-proposal.pdf;

[10] S. Agostinelli et al., Nucl. Instr. Meth. A506, 250 (2003).

[11] E. Farnea, private communication.; 